\title{
Comparisons between CRM and CCM PFC*
}

\author{
Weiping Zhang ${ }^{2}$, Wei Zhang ${ }^{2}$, Jianbo Yang ${ }^{1}$, Faris Al-Naemi ${ }^{1}$ \\ ${ }^{1}$ Materials and Engineering Research Institute (MERI), Sheffield Hallam University (SHU), Sheffield, UK \\ ${ }^{2}$ Lab of Green Power \& Energy System (GPES), North China University of Technology (NCUT), Beijing, China \\ Email: jumbo-yang@hotmail.com \\ Received September, 2012
}

\begin{abstract}
The paper presents detailed comparisons between CRM (critical conduction mode) and CCM (continuous conduction mode) control schemes used for Boost PFC converter. The two schemes are analyzed and compared under the chips of L6561 and UC 3854 which are commonly used for CRM and CCM respectively. Both schemes are based on multiplier; however, the CCM is more complex and needs more periphery components which increase the cost. The Boost PFC under CRM is easier to be implemented. Nevertheless, the variable switch frequency makes the system (including the power-stage inductor and capacitor) hard to design. It seems that the CRM PFC is more attractive in low power applications which only need to meet IEC61000-3-2 D standard. Some experiment results are also presented for the comparison.
\end{abstract}

Keywords: CRM; CCM; PFC; Boost

\section{Introduction}

In conventional AC-DC conversion, a capacitor following a bridge rectifier is used to derive DC voltage from the AC power source. With this capacitor, however, the input current pulsates. This pulsating current increases the input current harmonics and results in a low power factor less than 0.64[1]. To reduce the input current harmonics and increase the power factor, a high power factor technique is desired. Power factor correction is to make the input to a power supply look like a simple resistor. Therefore, an AC-DC converter based on power stage of boost configuration as shown in Figure 1 has been studied as a high-power-factor pre-regulation circuit [2].

As shown in Figure 1, according to the current of inductor L, the operation modes can be specified as: CCM (continuous conduction mode), DCM (discontinuous conduction mode and CRM (critical conduction mode). The critical conduction mode operates at the boundary of CCM and DCM.

In recent years, PFC with different control schemes has been proposed. Nevertheless, a thorough comparison is seldom reported.

In this paper, a detailed comparison between CCM and CRM with constant on time is presented including appli-

*Project supported by Natural Science foundation of China (N0. 51277004). The Importation and Development of High-Caliber Talents Project of Beijing Municipal Institutions (No.IDHT20130501) cation area, components selection and small signal analysis of the entire system. Some experiments are carried out for the experimental comparison. The experimental results match the theoretical analysis well.

\section{Comparison of Control}

\subsection{Control Schemes}

The control schemes of the two operating modes for the identical boost PFC converter are depicted in Figures 2 and 3 respectively. Figure 2 illustrates the boost PFC under CRM. Correspondingly, a boost PFC under CCM is detailed in Figure 3.

One point should be made clear that CRM and CCM are only concern with the minimum of the input inductor current. CRM means the input inductor current touches 0 without maintenance in every switching circle. While, the input inductor current is always above zero in CCM operating mode.

As depicted, PFC under CRM is actually the peak current control. The current is sensed from switch and compared with the programming current. When the input

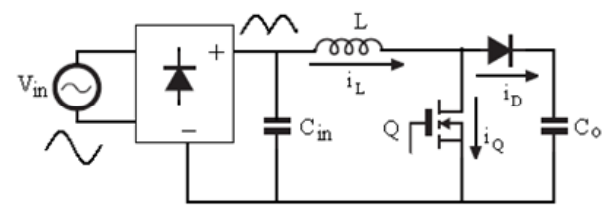

Figure 1. Boost PFC converter. 


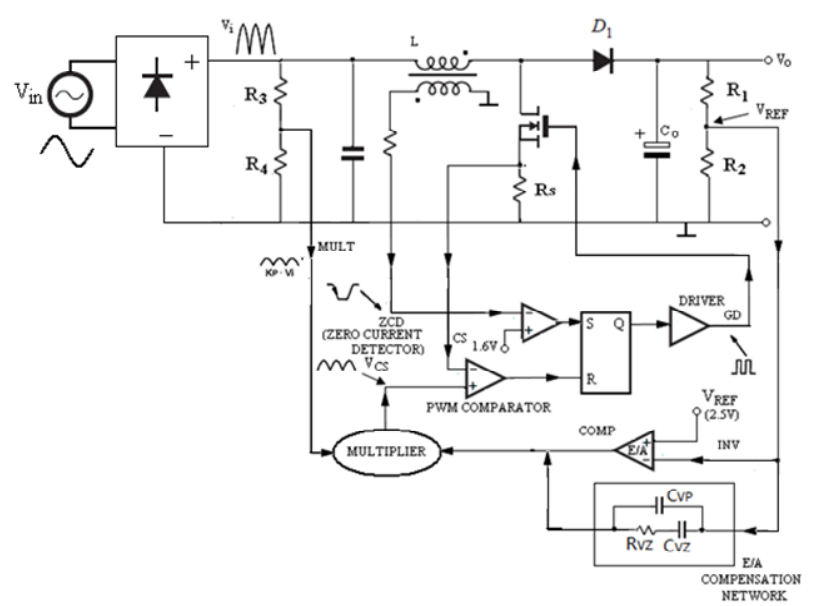

Figure 2. CRM (Critical Conduction Mode).

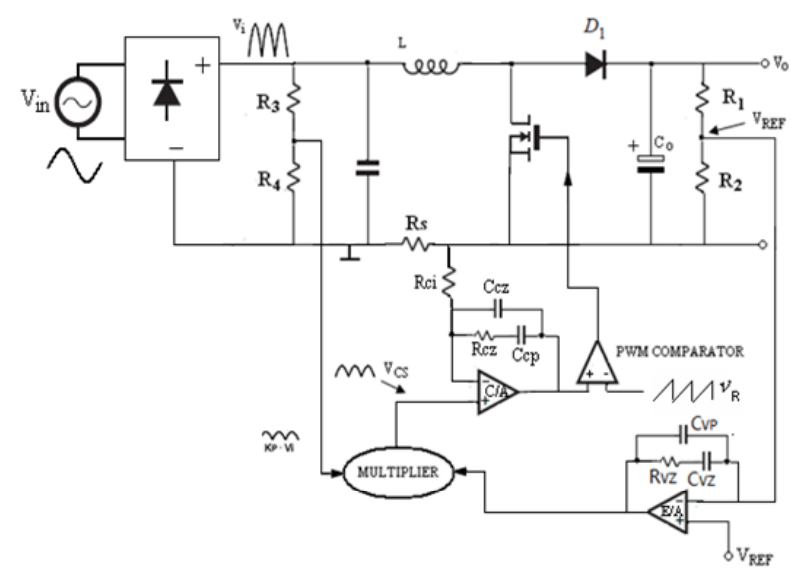

Figure 3. CCM (Continuous Conduction Mode).

current equals to the programming signal, the switch will be turned off. The switch turned on signal comes from a ZCD (zero current detection) function block. This ZCD block will send a turned on signal to switch when the input current reaches zero. Therefore, the input current will touch zero in every switch cycle. In contrast, the PFC under CCM is the average current control and has different configuration. The input current is sensed from inductor instead of the switch. This sensed current is still compared with the programming signal; however, a current error amplifier rather than a comparator is employed here. Thus, the error signal is amplified and compared with a PWM generator.

It is clear that there is one compensation network in CRM PFC; nevertheless, two compensators exist in the CCM PFC. More components are needed in the average current CCM control scheme. This increases the complexity and cost of the CCM average current control.

\subsection{Control Blocks}

According to Figures 2 and 3, the small signal block diagrams of the two implementations can be detailed as Figures 4 and $5[3,4]$. As shown, $G_{1}(s)$ is for the variations in the output of compensator to variations to the output of multiplier. $G_{2}(s)$ in Figure 4 is the ratio of output variations of the multiplier to the inductor current variations. $\mathrm{G}_{\mathrm{ci}}(\mathrm{s})$ in Figure 5 means the ratio of variations of input current to the variations of the output of current compensator. The means of other blocks are discussed in details in [4,5].

Obviously, there is one more loop in the CCM PFC under average current control. This increases the complexity. The inner loop should be decided before design the outer loop. However, as the input current is controlled directly, the distortions of the input current are much better than the CRM PFC.

The loop gain without the compensator of the both control scheme have just one pole [5,6]. This decreases the complexity of design of the compensator. The compensator can have the configurations and the frequency responses simulated by Matlab are presented in Figures 6 and 7.

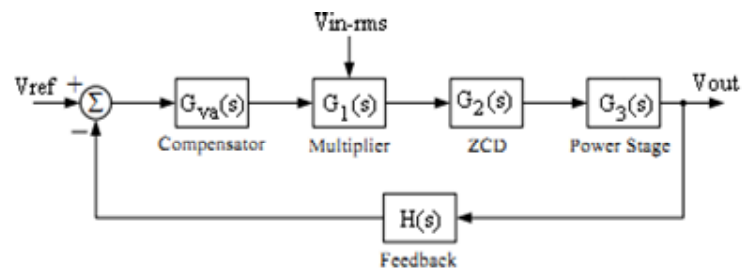

Figure 4. Control block of CRM.

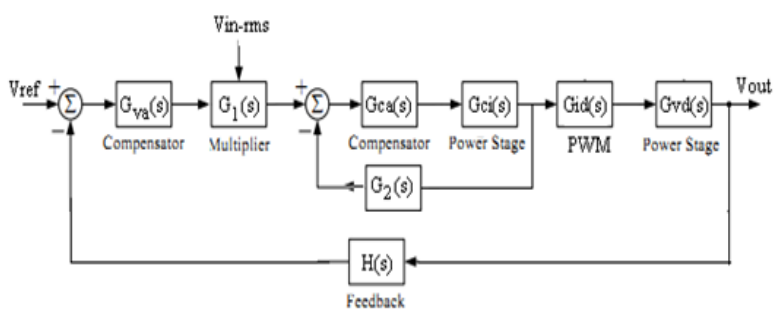

Figure 5. Control block of CCM.

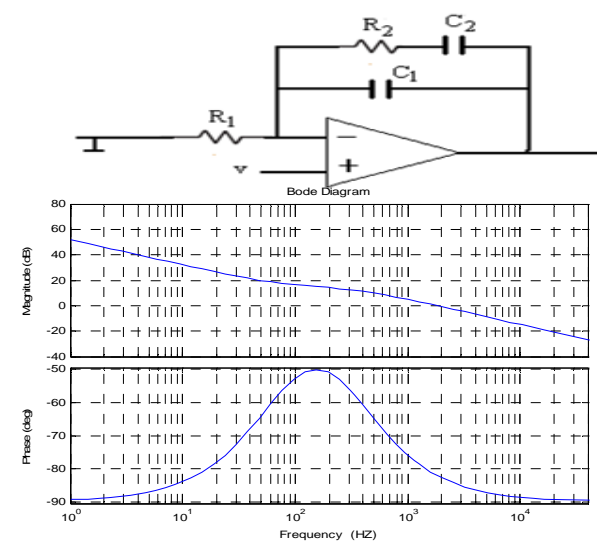

Figure 6. One-zero, two-pole compensator. 


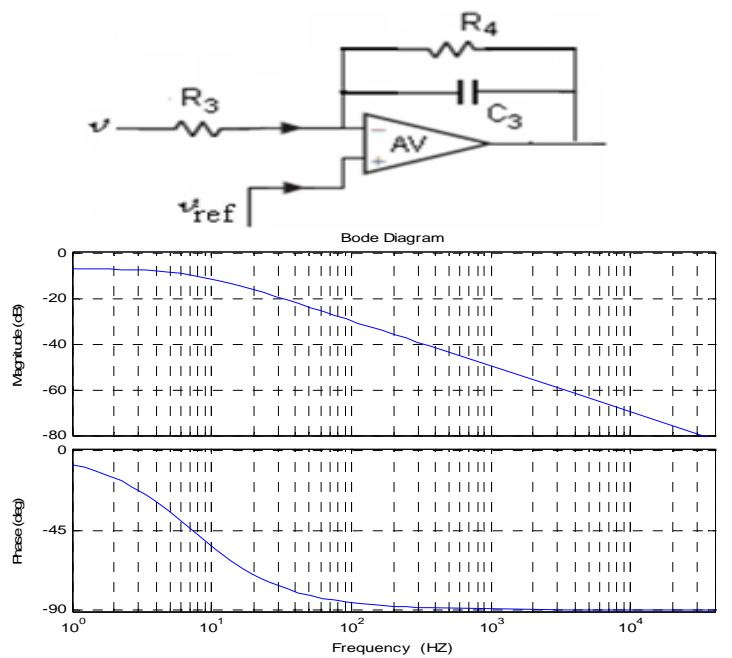

Figure 7. One-pole compensator.

Both of the compensators are suitable for the CRM and CCM PFC except that the CCM PFC with average current control has two loops and two compensators. This makes the CCM PFC more complicated to design. The CCM design process is detailed in [7]. The CRM $\mathrm{PFC}$ is designed in detail in [8].

\section{Comparison of Waveforms}

As discussed in section 2, the switch of CRM boost PFC is turned on when the inductor current reaches zero and turned off when the inductor current equals to the programming signal. Therefore, the envelope of the input current is the rectified AC line signal since the programming signal is derived from the rectified AC line voltage. Things turn to be different in the CCM PFC. As a large gain amplifier is implemented for the input current signal, the input current is forced to follow the programming signal which is also derived from the rectified AC line voltage. As a result, the average value of input current will follow the programming signal [7]. The inductor waveforms and duty circle are presented in Figures 8 and 9.

As shown, the peak of input current follows a rectified AC line signal under CRM PFC. Furthermore, the average input current equals to half of the programming signal. The average of the input current under CCM PFC equals to the programming signal. So the CRM PFC is not suitable for the high power applications.

For power applications higher than 300w, CCM PFC is widely used. For power applications lower than 300w, CRM PFC is more popular [10].

There are actually two constants in both implementations respectively. In CRM PFC, when switch is on:

$$
\frac{v_{i} T_{\text {on }}}{l} R_{s}=\frac{R_{4}}{R_{3}+R_{4}} k_{2} v_{i}=k_{1} k_{2} v_{i}
$$

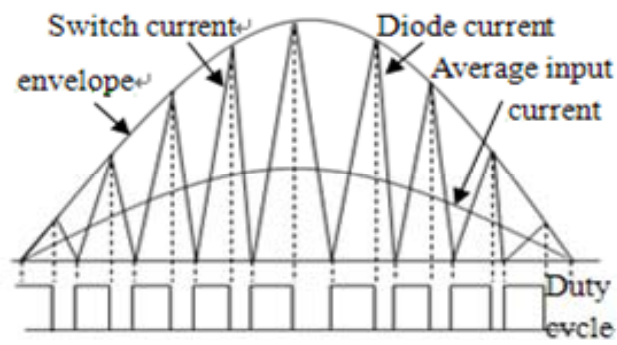

Figure 8. Input current of CRM.

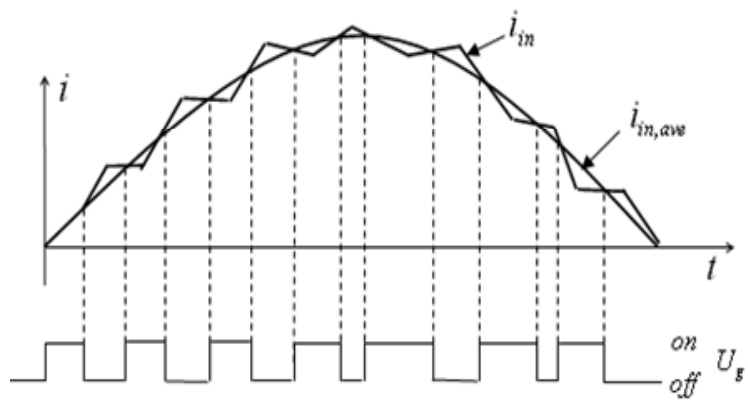

Figure 9. Input current of CCM.

where $\mathrm{Vi}$ is the input voltage, Rs is the sensing resistor, $l$ is the input inductor and R3, R4 refers to the Figure 2; $\mathrm{k}_{2}$ is output of voltage amplifier (E/A in Figure 2) which is not change under stable state. $T_{\text {on }}$ is the on-time of the switch. $k_{1}=\frac{R_{4}}{R_{3}+R_{4}}$, thus,

$$
T_{\text {on }}=\frac{k_{1} k_{2} l}{R_{s}}
$$

When the power stage circuit is determined, Ton is determined too. As a result the turned on time of switch of CRM PFC is constant. This is quite different with the CCM PFC. For the average current control with UC3854; the switch frequency is constant [9].

As for Boost PFC converter,

$$
\frac{V_{o}}{v_{\text {in }}}=\frac{1}{1-d}=\frac{1}{1-\frac{T_{o n}}{T}}
$$

Therefore, for CRM boost PFC, the switch frequency will be minimum when input voltage reaches its peak. The max frequency happens when the input voltage goes across zero.

The minimum switch frequency of the CRM PFC can be obtained as, [8]

$$
f_{\min }=\frac{1}{2 L P_{i n}} \frac{V_{i n, r m s}^{2}\left(V_{o}-\sqrt{2} V_{i n, r m s}\right)}{V_{o}}
$$

where $P_{\text {in }}$ is the input power, $V_{\text {in,rms }}$ is the RMS value of the input voltage, $V_{o}$ is the output voltage. The variable frequency will bring a little complexity in design of the 
circuit. This will be discussed next.

\section{Input Inductor Selection}

The selections of components of CCM PFC circuit have been provided in [7]. The approach of selections is almost the same with CRM PFC circuit except the input inductor (L in Figure 1). For CRM PFC, the minimum switch frequency dominates the design of the input inductor. Mathematically manipulating of (4), the input inductor can be obtained as.

$$
L_{\max }=\frac{1}{2 f_{\min } P_{i n}} \frac{V_{i n, \text { rms }}^{2}\left(V_{o}-\sqrt{2} V_{i n, \text { rms }}\right)}{V_{o}}
$$

$V_{i n, r m s}$ is the rms value of the low input line voltage. Such as $90 \mathrm{v}$ when the input voltage is required between $90-260 \mathrm{v}$. The input current distortion is the most important consideration for the design of input inductor of CCM boost PFC. When the power is constant, the input current will be higher for the low input voltage (for universal input range). Thus,

$$
I_{i n, \max }=\frac{\sqrt{2} P_{i n}}{V_{i n(\min ), r m s}}
$$

$I_{i n, \max }$ means the maximum input current. The peakto-peak ripple current is assumed to be $20 \%$ of the input current. Refers to (3), the input inductor can be obtained as,

$$
\begin{aligned}
L & =\frac{v_{\text {in(min), peak }} D}{f_{s} \Delta I} \\
& =\frac{2.5 v_{\text {in(min), peak }}^{2}\left(V_{o}-v_{\text {in(min), peak }}\right)}{f_{s} P_{i n} V_{o}}
\end{aligned}
$$

where $V_{\text {in(min),peak }}$ is the peak of the low input line voltage. $P_{i n}$ is the input power. $V_{o}$ is the output voltage and $f_{s}$ is the switching cycle.

As discussed, the selection of the inductor for CRM is mainly concerns the minimum switch frequency. Whereas, for CCM, the input inductor selects from the maximum peak-to-peak ripple current.

\section{Experimental Results}

The performances of the two implementations are verified with two 100w prototypes. The CRM PFC is carried out by L6561 and the CCM PFC is implemented with UC3854. The output voltage is set to be $360 \mathrm{v}$. The results are shown below. As shown in Figure 10, Both of the operating modes have almost the same power factor. The power factor of CCM is 0.98 which is a little higher than the one of CRM, which is 0.96 . The efficiency of CCM PFC is $84 \%$, while the efficiency of CRM PFC is about $80 \%$. As the input current of the input current operates in critical conduction mode, the current falls to zero once during every switching cycle as shown in Figure 11. This cause the current distortion of CRM is more

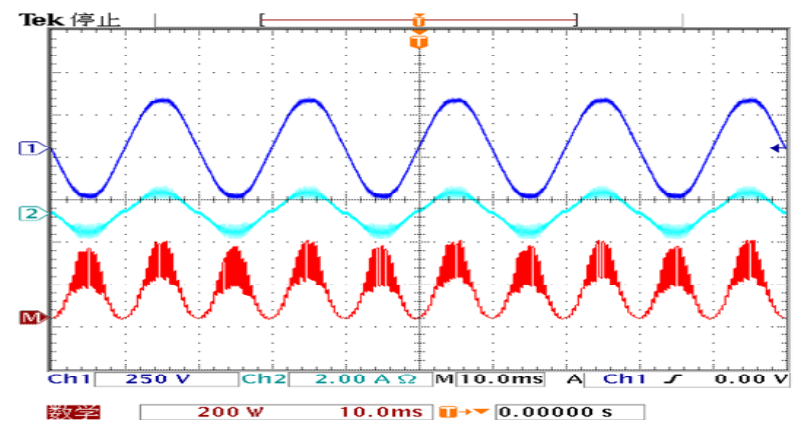

(a) CRM: input voltage: 220 vac; current: $0.57 \mathrm{~mA}$ (rms); power factor: 0.96

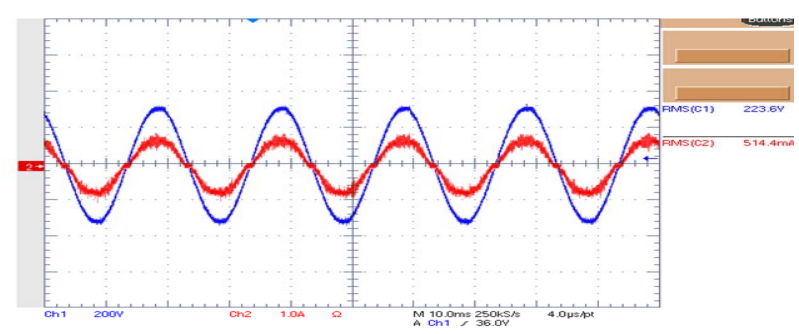

(b) CCM: input voltage: 220 vac; input current: $0.54 \mathrm{~mA}$ (rms); power factor: 0.98

Figure 10. Input waveforms.

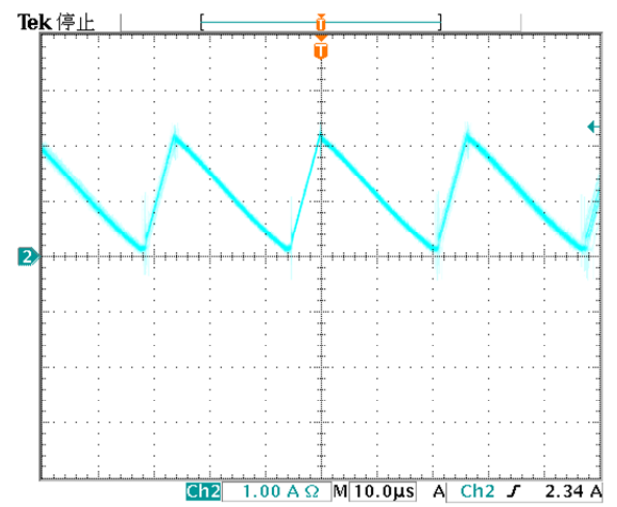

(a) Switching cycle

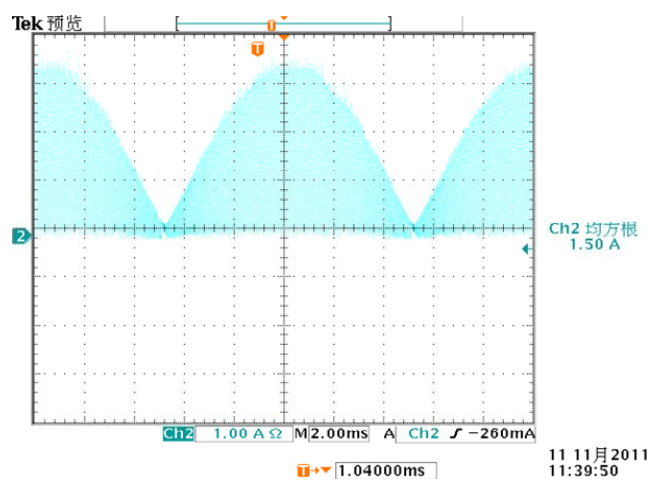

(b) Line frequency

Figure 11. Input inductor current of CRM ( $\mathrm{L}$ in Figure 2). 


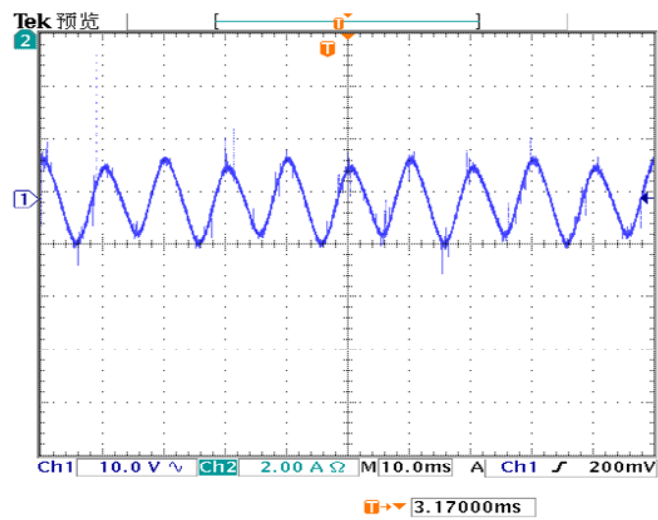

(a) CRM: $20 \mathrm{v}$ (peak-to-peak)

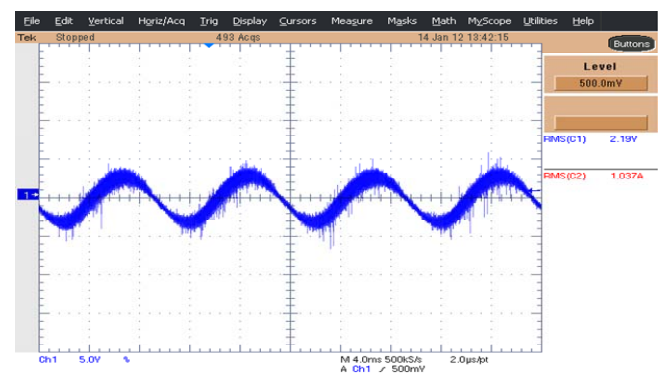

(b) CCM: $15 \mathrm{v}$ (peak-to-peak)

Figure 12. Output voltage ripple.

serious than the CCM PFC, which leads to a lower efficiency. Besides, the CRM PFC requires EMI filters to eliminate the high frequency harmonics of the input current which further reduces the efficiency and complicates the design.

Figure 12 shows the output voltage ripples. The peakto-peak value of the voltage ripple of CRM PFC is about $20 \mathrm{v}$, and the counterpart of CCM is about $15 \mathrm{v}$, which is $5 \mathrm{v}$ lower.

It seems that the CCM PFC is a better choice than CRM PFC with respect to the power factor and efficiency. However, there are two control loops which need to be design in the CCM PFC. This increase the complexity.

\section{Conclusions}

Boost PFC converters under CRM and CCM are compared with each other in this paper. Some conclusions can be obtained as:

1) The peak inductor current of the CRM PFC is twice higher than the average input current, which makes the CRM PFC more suitable for low power applications (lower than $300 \mathrm{w}$ ). The CCM PFC dominates the high power applications (higher than $300 \mathrm{w}$ ).

2) The inductor current of CRM PFC reaches zero during each switch cycle and only has a rectified sinusoidal envelope. Whereas, the inductor current is almost the same with the input current except some high harmonics.

3)CCM PFC has the constant switch frequency and variable turned-on time. While CRM PFC has constant switch turned-on time, variable switch frequency which makes the design of power stage components more complicated.

4) CRM PFC has only one control loop and needs fewer external components which is easy to be implemented. There are two control loops in the CCM PFC. the CCM PFC needs more external components which will increase the costs, however, the input current of CCM PFC has fewer harmonics and higher efficiency.

Both PFC operating modes have their own advantages and disadvantages. Selection is depending on the applications.

\section{REFERENCES}

[1] X. W. Mao and D. W. Zhu, "The Control IC, Principles and Applications of PFC,” 2007, 1st edition, China Electric Power PRESS

[2] R. Keller and G. Baker, "Unity Power Factor off-line Switching Power Supply," in IEEE INTELEC record, 1984, pp. 332-339.

[3] W. P. Zhang, F. Chen, X. S. Zhao and Y. C. Liu, “A Discrete Modeling for Power Factor Correction Circuit," PEDS 2009, pp. 160-163.

[4] K. I. HWu, C. H. Wu and C. F. Chuang, "Development of AC-DC Converter for Laboratory Power Amplifier," PEDS 2009, pp. 1534-1541.

[5] W. P. Zhang, "The Modeling and Control of Switch Converter,” M, first edition, 1996.

[6] J. Sun, R. M. Bass, "Modeling and Practical Design Issues for Average Current Control,” in Conf. Rec, APEC Fourteenth annual, 1999, pp. 980-986

[7] P. C. TODD, "UC3854 Controlled Power Factor Correction Circuit Design”, UNITRODE application note, U134

[8] S. L. Xie, L. F. Liu and J. B. Liu, "100 W Power Factor Correction of Basing On L6561," AIMSEC 2011, $2^{\text {nd }}$ conference, pp. 3599-3602.

[9] S. L. Liu and Y. G. Yan, "UC3854A/B Power Factor Boost Controllers Provide the Improvements to UC3854 and Power Limiting with Sinusoidal Input Current for PFC Front Ends,” Electronic Components Application, Vol. 3, No. 1, Mar 2001, pp. 31-36.

[10] J. W. Kim, S. M Choi and K. T Kim, "Variable On-time Control of the Critical Conduction Mode Boost Power Factor Correction Converter to Improve Zero-Crossing Distortion” PEDS 2005, pp. 1542-1546. 